\title{
Thoughts of Zhuang Li-Xing's Mind-Regulating Acupuncture Therapy for Treatment of Post-Stroke Depression
}

\author{
Jun He \\ Department of Acupuncture and Moxibustion Medicine, First Affiliated Hospital of Guangzhou University of Traditional Chinese Medicine, \\ Guangzhou, China
}

Email address:

hx120912@163.com

\section{To cite this article:}

Jun He. Thoughts of Zhuang Li-Xing's Mind-Regulating Acupuncture Therapy for Treatment of Post-Stroke Depression. International Journal of Chinese Medicine. Vol. 3, No. 3, 2019, pp. 48-51. doi: 10.11648/j.ijcm.20190303.13

Received: May 22, 2019; Accepted: June 24, 2019; Published: September 9, 2019

\begin{abstract}
PSD affected the rehabilitation of limb function in stroke patients seriously and it is necessary to adopt effective approaches to treat PSD in clinic to enhance life quality of patients with stroke. The theories and practices for the treatment of post-stroke depression (PSD) with mind-regulating acupuncture therapy by Professor Zhuang Lixing were summarized. Professor Zhuang who has been a doctor for more than 30 years and now is a highly ranked and famous Chinese medicine practitioner, and Dr. Zhuang is an expert in treating PSD by using the mind-regulating acupuncture therapy and this therapy has the exact curative effectiveness. The pathogenesis of PSD is due to the disorder of $q i$ flowing and internal retention of phlegm as well as the blood stasis caused by stroke, which result in the deficiency of nutrition and the failed shelter of spirit in brain. For improving the overall mental function of the patients with PSD, Professor Zhuang accurately locates the "spirit" and stimulates the optimal acupuncture points of Sishenzhen, Zhisanzhen, Yintang (EX-HN3), Shenmen (HT7), and Sanyinjiao (SP6) by electroacupuncture and points modification to induce $q i$ from the spirit and relieve the depression by activating $q i$ flow. Clinical practice of Professor Zhuang provides evidence for the treatment of PSD with acupuncture therapy.
\end{abstract}

Keywords: Post-Stroke Depression, Mind- Regulating Acupuncture Therapy, Zhuang Li-Xing, Sishenzhen, Zhisanzhen, Yintang (EX-HN3), Shenmen (HT7), Sanyinjiao (SP6)

\section{Introduction}

Post-stroke depression (PSD) is an affective disorder syndrome characterized by a series of depression and lack of interest in addition to the symptoms of stroke [1-2], with a combined incidence of PSD in the 5 years after stroke was $31 \%$, which seriously affected the rehabilitation of limb function in stroke patients. Therefore, it is necessary to take effective approaches to treat PSD in clinic to enhance life quality of patients with stroke. Now that many hospitals and general practitioners are adopting symptomatic drug therapy, and patients are prone to serious adverse reactions, such as cognitive impairment and slow response after long-term oral administration of drugs, with high-cost burden and poor patient compliance [3-4]. Acupuncture and moxibustion have unique advantages in treating PSD and the western medicine cannot match it [5-9]. For example, acupuncture and moxibustion therapy not only has the advantages of exact curative effect and few side effects, but also contributes to the further recovery of limb function in stroke patients. Among the multitudinous acupuncture and moxibustion therapies, the mind-regulating acupuncture therapy for treating PSD has been simplified and the exact curative effectiveness has been confirmed by clinical research [10].

Professor Zhuang Lixing, who had more than 30 years clinical experience, is a highly ranked and famous Chinese medicine practitioner in the Department of First Affiliated Hospital of Guangzhou University of Traditional Chinese Medicine (TCM) in Guangdong province. As the academic leader of the Traditional Jin's three needle Therapy's Inheritance Studio of the Chinese Medical School, Dr. Zhuang is an expert in treating the nervous system diseases, various pain syndromes and PSD by using the mind-regulating acupuncture therapy. This review mainly summarizes the 
mind-regulating acupuncture therapy for treating PSD practiced by Professor Zhuang.

\section{Pathogenesis of PSD in TCM}

Although there is no exact name for PSD, it is categorized to depressive syndrome in TCM according to the symptoms it causes. Many classical academic publications of TCM have relevant records of depressive syndrome, such as that when the organs of the body are vital, the human is healthy; while the body is depressed, the human is unhealthy. PSD not only has the characteristics of unsatisfactory emotional sensation and stagnation of vital energy, but also has the insufficiency of kidney and liver as well as $q i$ and blood stagnation, therefore TCM scholars believe that the affected region of PSD is mainly the brain, also involving the liver, kidney, heart and spleen; moreover its etiology and pathogenesis are asthenia in origin and superficiality. Intermingled deficiency and excess asthenia in origin are $q i$ deficiency and kidney deficiency; the asthenia in superficiality are $q i$ stagnation, blood stasis, and phlegm turbidity. In brief, the pathogenesis of PSD is due to the disorder of $q i$ flowing and internal retention of phlegm as well as blood stasis caused by the stroke, which results in the deficiency of nutrition and the failed shelter of spirit in brain [11].

\section{Treating and Curing Strategy of PSD in Chinese Medicine and Western Medicine}

Modern medicine believes that PSD, among the most frequent consequences of stroke, is an affective disorder syndrome. The pathogenesis is that lesions caused by stroke damage the emotion-related nerve center and interrupt the projections ascending from midbrain and brainstem, leading to a decreased bioavailability of the biogenic amines serotonin (5HT), dopamine (DA) and norepinephrine (NE) [12-13]. Currently, symptomatic pharmacotherapy used to treat PSD includes selective 5-HT reuptake inhibitors (SSRIs) [14-16], such as fluoxetine, paroxetine, sertraline, but these treatments tend to cause undesirable side effects. However, its depession risk, prolonged duration, and relatively high cost result in poor patient compiance. Consequenty, compementary and alternative medicine treatments have been sought, especially traditional Chinese medicine (TCM).

TCM management of health can be characterized as holistic with the emphasis on regulating the integrity of the human bodily functions and the interaction between individuals and their environments. Acupuncture has been clinically proved to own unique advantages in the treatment of PSD. Current researches suggest that the main affected region of PSD is the brain and the PSD affects mood through the brain network of the prefrontal-limbic circuit. The dysfunction of the brain, and the inharmony of the five internal organs (heart, liver, spleen, lungs and kidneys) affect the organism's spirit, will, emotion, and other activities, then ultimately result in the depression syndrome. Clinical studies [10] confirmed that the therapeutic effect of the mind-regulating acupuncture therapy on PSD, which focuses on adjusting brain function [17] is comparable to oral antidepressants, with the mood symptoms significantly improved. Moreover, the effects of mind-regulating acupuncture therapy may even extend beyond mood symptoms, e.g., to motor recovery, and thus significantly increase the proportion of patients reaching partial or full independence. The scores of TCM syndrome scale and Modified Edinburgh-Scandinavian Stroke Scale (MESSS) after acupuncture treatment are lower than those of antidepressants [10]. In addition, the incidence of adverse reaction and events of the mind-regulating acupuncture therapy was very low, making the compliance of patients better. Taken together, all of these demonstrate that the mind-regulating acupuncture therapy has great advantages over pharmaceutical therapy in treating PSD.

\section{Professor Zhuang's Clinical Experience in Treating PSD with Mind-Regulating Acupuncture Therapy}

Through mobilizing the qi-blood circulation to achieve the therapeutic purpose, mind-regulating acupuncture therapy practiced by Professor Zhuang is of great importance for regulating emotional factors, especially for the mental disease. The mind-regulating acupuncture therapy, made up the regulating the mind acupoints group, which not only can direct dialectical addition and subtraction according to the specific conditions of patients, but also simplified and has the exact curative effectiveness. PSD is classified to depressive syndrome which belongs to mental diseases in TCM. And the pathogenesis of PSD is due to the deficiency of nutrition and the failed shelter of spirit in the brain. Thus may concentrate on adjusting spirit function. Professor Zhuang accurately locates the "spirit" and stimulates the optimal acupuncture points of Sishenzhen, Zhisanzhen, Yintang (EX-HN3), Shenmen (HT7), and Sanyinjiao (SP6) by electroacupuncture and points modification to induce $q i$ from the spirit and relieve the depression by activating $q i$ flow.

Acupuncture and moxibustion emphasizes that the intensity of acupuncture must reach a threshold to generate deqi, which is necessary to achieve the best therapeutic effect. Deqi is an internal compound sensation of soreness, tingling, fullness, aching, cool, warmth and heaviness, and a radiating sensation at and around the acupoints. Respectively, Professor Zhuang thinks highly of deqi during acupuncture, and deqi is obtained via twisting and lifting achieved by acunpuncturist. Clinical studies have demonstrated that scalp acupuncture stimulation can regulate the electrical activity of the brain as well as the release of neurotransmitters with electroacupuncture [18]. Therefore, the therapeutic effect of the mind-regulating acupuncture therapy on PSD, which focuses on adjusting brain function by promoting the brain flow of blood and $q i$, using directional wave. 
Professor Zhuang accurately stimulates optimal acupuncture points of Sishenzhen, Zhisanzhen, Yintang (EX-HN3), Shenmen (HT7), and Sanyinjiao (SP6). Sishenzhen, a group of four acupoints each located anterior, posterior and lateral $1.5 \mathrm{cun}$ from DU20 which is the sea of marrow and has the effect of descending or raising the yang. Sishenzhen has the same effect as DU20, moreover, it can enhance the curative effect. The Sishenzhen radiation range is larger than DU20, because it's expanding the stimulation of the brain projection area [19]. Zhisanzhen consists of Benshen (GB13) and Shenting (DU24). Those acupoints play regulating the mind effect by calming the spirit and clearing pathogenic factors from the brain. Zhisanzhen can be used to cure emotional disorders, because it's situated in the scalp of the frontal lobe of the brain which is the center of emotion and intelligence [20]. Yintang (EX-HN3) belongs to Governor Meridian, and it works by stimulating the Governor Meridian to open the body's channel and calming the spirit. Electroacupuncture at Yintang can increase the content of 5-HT in the hypothalamus [21]. Shenmen (HT7), the Heart Meridian yuan-source acupoint, regulating and strengthening the heart, calming spirit as well as clearing heat from the heart channel. Sanyinjiao (SP6) located in Spleen Channel meeting with the Liver and Kidney Channels, which calming the spirit by nourishing the blood and yin, as well as fortifying the spleen and the stomach. A Clinical study indicated that electroacupuncture Shenmen and Sanyinjiao specificity activated the brain areas, especially frontal lobe 9, 10,11 areas, which closely related to intelligence and spiritual activities [22]. In a word, mind-regulating acupuncture therapy stimulates the optimal regulating-mind acupuncture points and makes effective by improving the overall spirit function.

\section{Conclusions}

PSD affected the rehabilitation of limb function in stroke patients seriously and it is necessary to adopt effective approaches to treat PSD in clinic to enhance life quality of patients with stroke [23]. Mind-Regulating acupuncture therapy formed by Professor Zhuang who has been a doctor for more than 30 years and now is a highly ranked and famous Chinese medicine practitioner. Dr. Zhuang is an expert in treating PSD by using the mind-regulating acupuncture therapy and this therapy has the exact curative effectiveness. Mind-regulating acupuncture therapy stimulates the optimal acupuncture points of Sishenzhen, Zhisanzhen, Yintang (EX-HN3), Shenmen (HT7), and Sanyinjiao (SP6) by electroacupuncture. Moreover, it is mainly arousing spiritual $q i$ as well as activating $q i$ circulation to relieve the depression. Clinical practice of Professor Zhuang provides evidence for the treatment of PSD with acupuncture therapy.

\section{Acknowledgements}

Funding: LIXING ZHUANG'S inheritance studio of TCM in Guangdong Province. Item No: Guangdong TCM Office [2018]. 5.

\section{References}

[1] Shaoshi W, Xinyu Z, Chunyan Z. Chinese Expert Consensus on Clinical Practice of Post-stroke Depression [J]. Chinese Journal of Stroke, 2016, 11 (8): 685.

[2] Hackett M L, Pickles K. Part I: frequency of depression after stroke: an updated systematic review and meta-analsis of observational studies [J]. Int J Stroke, 2014, 9 (8): 1017.

[3] Shuchang S. Clinical Research Progress of Acupuncture in the Treatment of Post-stroke Depression [J]. Chinese Medical Emergency, 2014, 23 (9): 1689.

[4] Lijun Y, Zhaoqing L, Jin Z, et al. Clinical study on intervention of Tongdu Tiaoshen acupuncture combined with cognitive therapy in patients with post-stroke depression [J]. World science and technology: modernization of traditional Chinese medicine, 2017, 19 (8): 1319.

[5] Mengmeng Y. Clinical Study on the Treatment of Post-Stroke Depression by Acupuncture Method of "Regulating Mind and Resolving Depression" [D]. Harbin: Heilongjiang University of Traditional Chinese Medicine, 2016.

[6] D M, Feng T. The mechanism and clinical research progress of electro-acupuncture in the treatment of post-stroke depression [J]. Chinese medicine emergencies, 2014, 23 (3): 476.

[7] Qianqian C, Shengchao C, Yunyan C, et al. Effects of Qi-regulating and Shen-regulating acupuncture on the expression of CREB and PDE4 mRNA in left anterior cortex and hippocampus of rats with post-stroke depression [J]. Journal of Anhui University of Traditional Chinese Medicine, 2014, 33 (4): 55.

[8] Haibing X. Effects of electroacupuncture at "Siguan" group points on NE, DA and 5-HT in different brain regions of PSD model rats [D]. Changsha: Hunan University of Traditional Chinese Medicine, 2015.

[9] Yang Y. Clinical Observation on Post-stroke Depression Treated by Acupuncture Method of Regulating Mind and Regulating Qi [D]. Harbin: Heilongjiang University of Traditional Chinese Medicine, 2013.

[10] Qisong L. Clinical Observation on the Treatment of Post-stroke Depression by Tiaoshen Acupuncture [D]. Guangzhou: Guangzhou University of Traditional Chinese Medicine, 2014.

[11] Weijun D, Daojun X. Progress in TCM treatment of post-stroke depression [J]. Journal of Clinical Chinese Medicine, 2013, 25 (11): 1042 .

[12] Xiuhua B, Yun S, Zhu Q. Progress in the pathogenesis of depression [J]. Yunnan Journal of Traditional Chinese Medicine, 2012, 33 (1): 64.

[13] Wei X, Xianbao Z, Zhen W, et al. Effects of Acupuncture on Behavior and Neurotransmitters in Hippocampus of Rats with Post-stroke Depression [J]. Shanghai Journal of Acupuncture and Moxibustion, 2017, 36 (6): 751.

[14] Xiaoyi H, Ying Z, Zehuai W. Rationality Evaluation of the Selection of Control Measures and Effect Indicators in Randomized Controlled Trial of Acupuncture and Moxibustion for Post-stroke Depression [J]. Journal of Guangzhou University of Traditional Chinese Medicine, 2015, 32 (3): 542. 
[15] Xiyan L, Wei Z, Qincong Z, et al. Treatment and prevention of post-stroke depression [J]. Chinese Journal of Gerontology, 2018, 38 (4): 1010.

[16] Qiqi Z, Junpeng G, Dingfang C. Drug therapy for post-stroke depression - past, present and future [J]. Journal of Fudan (Medical Edition), 2011, 38 (2): 178.

[17] Ping W, Bailu C, Qi P. Acupuncture and moxibustion in the treatment of post-stroke depression $[\mathrm{J}]$. Liaoning Journal of Traditional Chinese Medicine, 2015, 42 (5): 1132.

[18] Jiayin Z, Baoen H, Ruohua B. Effect of Acupuncture "Deqi" on EEG [J]. Shanghai Journal of Traditional Chinese Medicine, 1963, 8 (3): 21.

[19] Shenghu Z, Yuntian W, Jinrong L, et al. Clinical Study on the Treatment of Perimenopausal Depression with "Four Shen Acupuncture" Long-term Retention Acupuncture [J]. Liaoning Journal of Traditional Chinese Medicine, 2010, 37 (4): 726.
[20] Tzu-qi CH, Chao-rong W. Chinese Acupuncture and Moxibustion [J]. 2004, 24 (11): 62.

[21] Jianbin ZH, Lingling W. Advances in the mechanism of acupuncture in the treatment of depression [J]. Shanghai Journal of Acupuncture and Moxibustion, 2006, 25 (12): 44.

[22] Jin Z, Dayan Y, Xiao C, et al. Brain fMRI Observation of Insomnia Treated by Electro-Acupuncture at Shenmen and Sanyinjiao Points $[\mathrm{J}]$. Chinese Interventional Imaging and Therapy, 2011, 8 (3): 204.

[23] Flaster M, Sharma A, Rao M. Poststroke depression: A review emphasizing the role of prophylactic treatment and synergy with treatment for motor recovery [J]. Top Stroke Rehabil, 2013, 20 (2): 139. 